\title{
Bacterial Aggregation and Leukocytes Phagocytosing Bacteria in the Diagnosis of Catheter-related Bloodstream Infection
}

\author{
Misa Tokunaga ${ }^{1 \star}$, Sumiyo Miyakawa ${ }^{1}$, Nao Mae ${ }^{1}$, Takayuki Kamiya ${ }^{1}$, Ayako Sato ${ }^{1}$, Masaaki Yamada ${ }^{1}$, \\ Hidenobu Matsumoto ${ }^{1}$, Emiko Sato ${ }^{1}$, Masashi Ohe ${ }^{2}$, Ken Furuya ${ }^{2}$
}

\begin{abstract}
${ }^{1}$ Department of Laboratory Medicine, JCHO Hokkaido Hospital, JAPAN
${ }^{2}$ Department of Internal Medicine, JCHO Hokkaido Hospital, JAPAN

*Corresponding Author: 0625asim@gmail.com
\end{abstract}

Citation: Tokunaga M, Miyakawa S, Mae N, Kamiya T, Sato A, Yamada M, Matsumoto H, Sato E, Ohe M, Furuya K. Bacterial Aggregation and Leukocytes Phagocytosing Bacteria in the Diagnosis of Catheter-related Bloodstream Infection. Electron J Gen Med. 2020;17(3):em201. https://doi.org/10.29333/ejgm/7849

A 96-year-old man with Alzheimer's disease and urinary tract infection (UTI) was admitted to our hospital for totally implantable central venous access device implantation. Medication and nutritional solutions were provided to the patient via the central venous catheter (CVC) because he had difficulty in swallowing food and water. He was treated with levofloxacin (LEV) for the UTI. Although urinalysis improved, fever continued. The patient exhibited violent behavior; therefore, the blood samples were taken via the CVC, rather than by venipuncture. Ten days after initiating LEV, laboratory findings indicated a white blood cell count of $7,510 / \mu \mathrm{L}$ (neutrophils, $70.0 \%$ ), and C-reactive protein levels of 7.44 $\mathrm{mg} / \mathrm{dL}$. We observed tentatively the blood specimen under the microscope. A peripheral blood smear revealed bacterial aggregation and leukocytes (i.e., neutrophils and monocytes) phagocytosing bacteria (Figure 1A, B, C, D). The presence of these in the peripheral blood smear, immediately raised suspicions of catheter-related bloodstream infection (CRBSI), resulting in prompt catheter removal. On the same day, further blood samples were obtained by venipuncture. In this case, however, the peripheral blood smear revealed neither bacterial aggregation, nor leukocytes phagocytosing bacteria. Methicillin-resistant Staphylococcus epidermis bacteria were detected in blood samples obtained via the CVC, after $3.6 \mathrm{~h}$ of both aerobic and anaerobic culturing, whereas blood samples obtained by venipuncture required $20.1 \mathrm{~h}$ of aerobic culturing, and $21.2 \mathrm{~h}$ of anaerobic culturing before the same bacteria were detected. Based on these findings, the patient was diagnosed as having CRBSI. He was successfully treated with vancomycin. CRBSI is defined as the presence of bacteremia originating from an intravenous catheter. In situations where the catheter cannot be withdrawn, diagnosis of CRBSI requires separate blood cultures to be obtained simultaneously from the catheter lumen and from peripheral veins. Definitive diagnosis depends on these samples being shown to meet the criteria for CRBSI in quantitative blood cultures (i.e., a colony count three times greater in the sample drawn through a catheter than in the sample drawn from the peripheral vein). Alternatively, differential time to positivity may be used to confirm diagnosis, in which case, positivity of blood cultures obtained through the catheter should be evident $\geq 120 \mathrm{~min}$ before those obtained from a peripheral vein (1). In view of potential contamination, blood culture specimen collection from intravascular indwelling catheters is generally not recommended for the diagnosis of catheter-unrelated bloodstream infections. However, specimen collection from the catheter and catheter tip culture is strongly recommended for the diagnosis of CRBSI (2). In such cases, microscopic examination of blood smears obtained from the catheter may provide useful clinical evidence to raise immediate suspicion of CRBSI. 


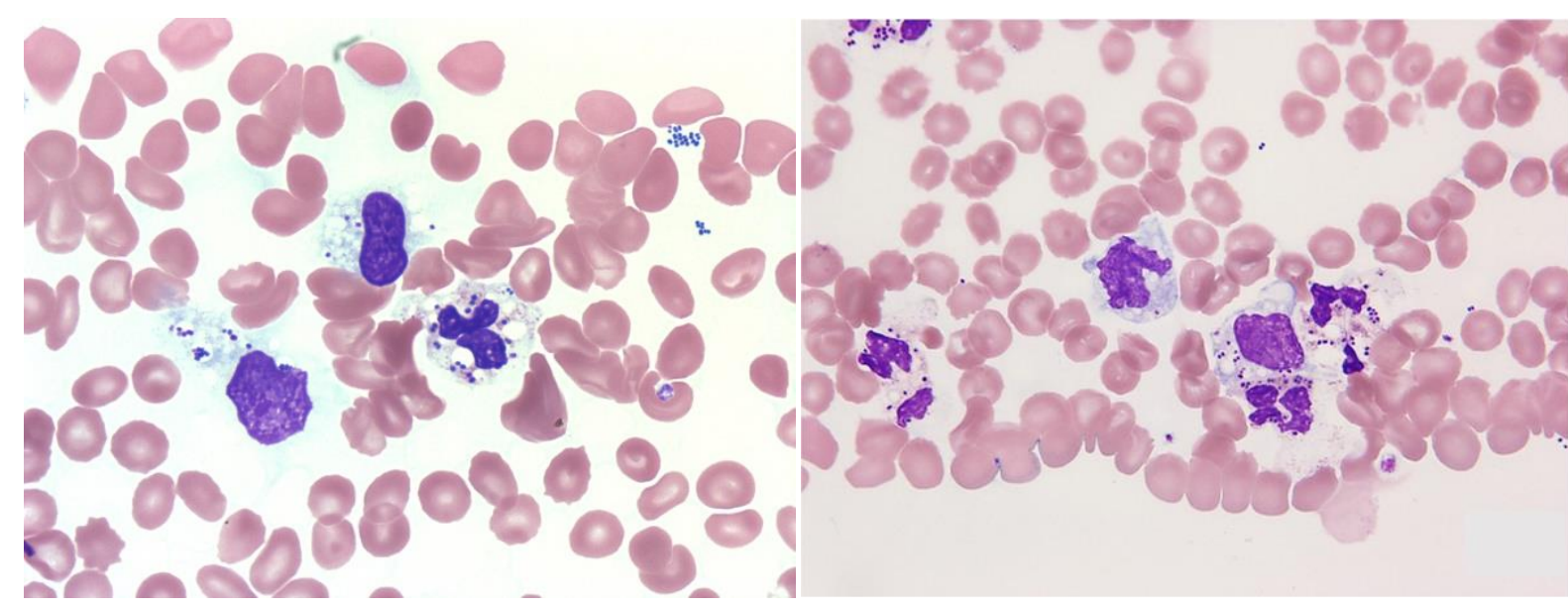

(A)

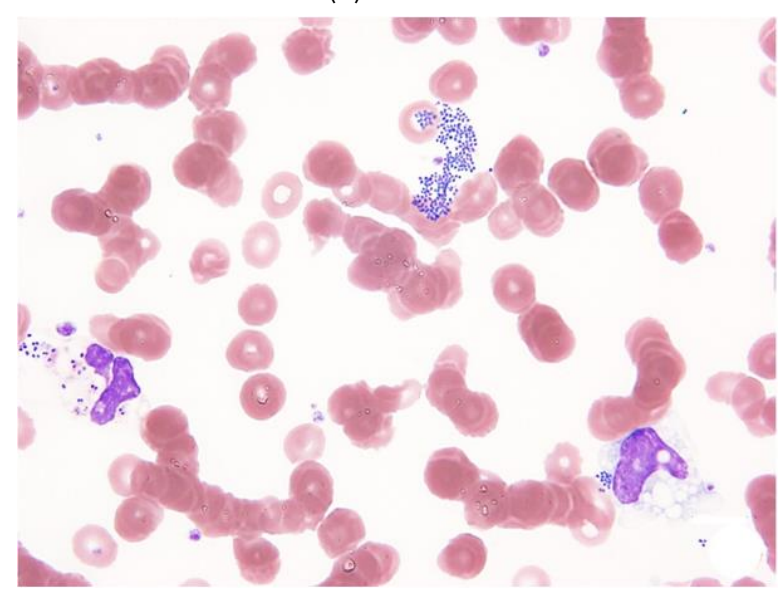

(C)
(B)

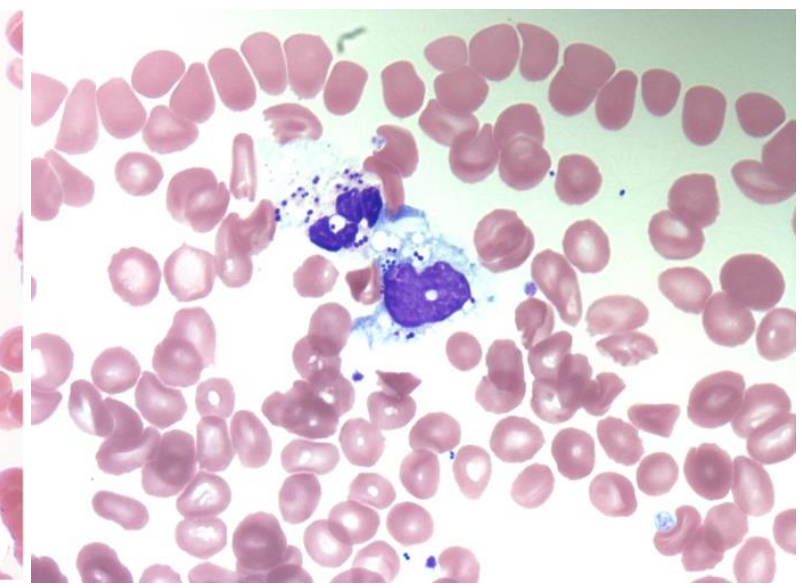

(D)

Figure 1. (A) (B) (C) (D) The peripheral blood smear reveals bacterial aggregation and leukocytes (i.e., neutrophils and monocytes) phagocytosing bacteria $(\times 1,000$, Wright-Giemsa stain)

\section{REFERENCES}

1. Canton-Bulnes ML, Garnacho-Montero J. Practical approach to the management of catheter-related bloodstream infection. Rev Esp Quimoiter. 2019;32(suppl 2):38-41.
2. DesJardin JA, Falagas ME, Ruthazer R, Griffith J, Wawrose $D$, Schenkein D, et al. Clinical utility of blood cultures drawn from indwelling central venous catheters in hospitalized patients with cancer. Am Intern Med 1999;131:641-7. 